\title{
The Efficacy of Hypotensive Agents on Intraoperative Bleeding and Recovery Following General Anesthesia for Nasal Surgery: A Network Meta-Analysis
}

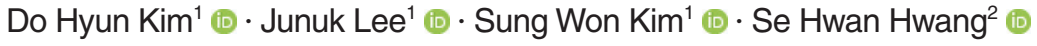 \\ ${ }^{1}$ Department of Otolaryngology-Head and Neck Surgery, Seoul St. Mary's Hospital, College of Medicine, The Catholic University of Korea, Seoul; \\ ${ }^{2}$ Department of Otolaryngology-Head and Neck Surgery, Bucheon St. Mary's Hospital, College of Medicine, The Catholic University of Korea,
}

Seoul, Korea

Objectives. A systematic review of the literature was conducted to evaluate hypotensive agents in terms of their adverse effects and associations with perioperative morbidity in patients undergoing nasal surgery.

Methods. Two authors independently searched databases (Medline, Scopus, and Cochrane databases) up to February 2020 for randomized controlled trials comparing the perioperative administration of a hypotensive agent with a placebo or other agent. The outcomes of interest for this analysis were intraoperative morbidity, operative time, intraoperative bleeding, hypotension, postoperative nausea/vomiting, and postoperative pain. Both a standard pairwise meta-analysis and network meta-analysis were conducted.

Results. Our analysis was based on 37 trials. Treatment networks consisting of six interventions (placebo, clonidine, dexmedetomidine, beta-blockers, opioids, and nitroglycerine) were defined for the network meta-analysis. Dexmedetomidine resulted in the greatest differences in intraoperative bleeding $(-0.971 ; 95 \%$ confidence interval $[\mathrm{CI}],-1.161$ to $-0.781)$, intraoperative fentanyl administration ( $-3.683 ; 95 \% \mathrm{CI},-4.848$ to -2.518$)$, and postoperative pain ( -2.065 ; $95 \% \mathrm{CI},-3.170$ to -0.960 ) compared with placebo. The greatest difference in operative time compared with placebo was achieved with clonidine $(-0.699 ; 95 \% \mathrm{CI},-0.977$ to -0.421$)$. All other agents also had beneficial effects on the measured outcomes. Dexmedetomidine was less likely than other agents to cause adverse effects.

Conclusion. This study demonstrated the superiority of the systemic use of dexmedetomidine as a perioperative hypotensive agent compared with the other five tested agents. However, the other agents were also superior to placebo in improving operative time, intraoperative bleeding, and postoperative pain.

Keywords. Antihypertensive Agents; Nasal Surgical Procedures; Morbidity; Controlled Hypotension

\section{INTRODUCTION}

Regardless of whether nasal surgery, such as septoplasty and endoscopic sinus surgery, is performed under local or general anes-

\footnotetext{
- Received April 9, 2020

Revised May 27, 2020

Accepted May 27, 2020

- Corresponding author: Se Hwan Hwang

Department of Otolaryngology-Head and Neck Surgery, Bucheon St.

Mary's Hospital, College of Medicine, The Catholic University of Korea,

327 Sosa-ro, Wonmi-gu, Bucheon 14647, Korea

Tel: +82-32-340-7044, Fax: +82-32-340-2674

E-mail: yellobird@catholic.ac.kr
}

thesia [1], intraoperative bleeding can cause difficulties with airway management [2]. To prevent airway problems during nasal surgery, general anesthesia is preferred [3], although it can lower capillary resistance and thereby increase the bleeding risk [4]. In addition, the medications used for general anesthesia can change vascular elasticity and cardiovascular reflexes, such as peripheral vasodilation, while decreasing the activity of the sympathetic nervous system. Previous in vivo studies showed a decrease in vascular resistance in patients under general anesthetics [5].

Bleeding during otorhinolaryngologic surgery reduces intraoperative visibility, which greatly hinders the procedure. Several agents, such as beta-blockers, alpha-blockers, and opioids, can

Copyright @ 2021 by Korean Society of Otorhinolaryngology-Head and Neck Surgery.

This is an open-access article distributed under the terms of the Creative Commons Attribution Non-Commercial License (https://creativecommons.org/licenses/by-nc/4.0)

which permits unrestricted non-commercial use, distribution, and reproduction in any medium, provided the original work is properly cited. 
be used to maintain a low intraoperative blood pressure, thereby reducing intraoperative blood loss and improving visualization of the surgical field [6,7]. Traditional meta-analyses of the effects of individual agents on blood loss have been conducted, but they were inherently limited to direct pairwise comparisons between a single treatment and a single alternative, whereas multiple treatment options and the ranking thereof were methodologically not possible. In contrast, a network meta-analysis (NMA) can be used to compare multiple treatment options simultaneously, as it combines all direct and indirect evidence from randomized controlled trials (RCTs). Moreover, an NMA provides a ranking of the assessed treatment options, thus allowing clinicians to choose the most effective treatment [8]. In this review, we present the results of our NMA of the efficacy of six different hypotensive agents used during nasal surgery. The findings can simplify evidence-based clinical decision-making in the management of patients undergoing nasal surgery.

\section{MATERIALS AND METHODS}

\section{Search strategy and selection of studies}

The search strategy was designed and reviewed by a clinical librarian, who has been an information specialist for more than 10 years. And the searching of the database such as Medline, Scopus, the Cochrane Controlled Register of Trials, and google scholar were performed in February 2020. We also tracked the reference lists of included studies and checked the existing systematic reviews to find relevant studies. Parts of the strategies are listed in Supplementary Tables 1-3, and diagram of the study selection process was presented in Fig. 1. The duplicated and apparently irrelevant studies were excluded through screening titles and abstracts by two reviewers (DHK, SHH) independently, and full texts of potentially eligible articles were assessed for inclusion independently by two reviewers once more. Any discrepancy in the selection of literature was resolved by consensus or the third author.

All relevant RCTs were included for minimizing the influence of the population characteristics such as distribution of age, sex, or severity of nasal disease status in the individual studies. All enrolled studies evaluated the hypotensive agents in terms of

\section{H I G H L L I G G H T S}

- Bleeding during otorhinolaryngologic surgery reduces intraoperative visibility, which greatly hinders the procedure.

- Dexmedetomidine showed promise as a perioperative hypotensive agent.

- Clonidine, dexmedetomidine, beta-blockers, opioids, and nitroglycerine improved operative time, intraoperative bleeding, and postoperative pain.

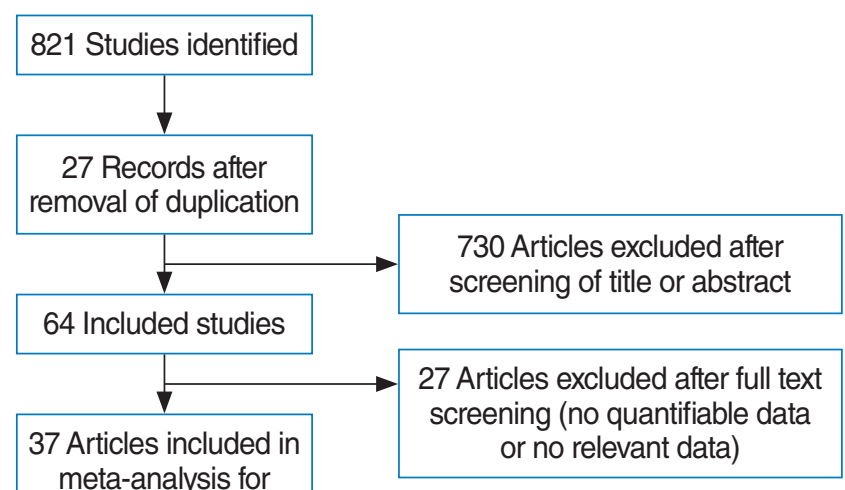

Fig. 1. Diagram of the study selection process.

their adverse effects and induction of perioperative morbidity in patients undergoing nasal surgery. In terms of participants, only adult-patients (more than 18 years old) undergoing nasal surgery including mainly endoscopic sinus surgery for chronic rhinosinusitis as well as septoplasty and rhinoplasty were included, and the physical condition of enrolled patients was in American Society of Anesthesiologists class I (healthy) or II (mild systemic disease). Patients with cardiovascular diseases, patients receiving cardiovascular active drugs, and patients receiving drugs influencing blood coagulation were excluded. Missing or incomplete data in the included studies were directly obtained from the authors. This NMA is reported based on the Preferred Reporting Items for Systematic Reviews and Meta-analyses for NMA.

\section{Data extraction and risk of bias assessment}

Data from the included studies were extracted using standardized forms and independently examined by the two authors. The evaluated outcomes consisted of operative time [1,3,6,9-35], mean intraoperative bleeding $[1,6,9,12-15,17,22,25-27,33,34$, $36,37]$, dose of intraoperative fentanyl administered [1,6,13,15, $19,22,30,32,38]$, postoperative pain $[3,7,10,11,22,33,36,39]$, the occurrence of postoperative nausea and vomiting (incidence or percentage of patients) $[3,7,10,11,13,19,22,27,29,31,33,39$, $40]$, and intraoperative hypotension $[3,11,13,22,27,32,35,36$, $39,41]$. The outcomes were compared with respect to the other treatment strategies and the control groups. Data were extracted from the included studies regarding patient number, grading scale, amount of analgesics, incidence of adverse effects, and $P$ values for comparisons of the various agents and respective patient groups. The risk of bias for each study was evaluated using the Cochrane risk of bias tool.

\section{Statistical analysis}

The meta-analysis was performed using R 3.5.0 netmeta package (R Foundation for Statistical Computing, Vienna, Austria). A random-effects NMA within a frequentist framework [42] was conducted to achieve combined results in the form of standard- 
Table 1. Summary of the studies included in the network meta-analysis

\begin{tabular}{|c|c|c|c|c|c|}
\hline Study & $\begin{array}{c}\text { Patient (male:female)/ } \\
\text { age (yr) }\end{array}$ & $\begin{array}{c}\text { ENT operation/ } \\
\text { underlying disease }\end{array}$ & Comparison & Outcome measure analyzed & $\begin{array}{l}\text { Risk of bias of } \\
\text { randomized studies }\end{array}$ \\
\hline $\begin{array}{l}\text { Lee et al. } \\
\text { (2013) [35] }\end{array}$ & $66(34: 32) / 45.5 \pm 12.1$ & $\begin{array}{l}\text { ESS/no cardiovascular } \\
\text { disease }\end{array}$ & $\begin{array}{l}\text { Dexmedetomidine vs. } \\
\text { remifentanil }\end{array}$ & $\begin{array}{l}\text { Surgical time } \\
\text { Adverse effects (hypotension) }\end{array}$ & Low risk of bias \\
\hline $\begin{array}{l}\text { Polat et al. } \\
\text { (2015) [39] }\end{array}$ & $\begin{array}{l}90(60: 30) / 36 \text { (range, } \\
\text { 23-53) }\end{array}$ & $\begin{array}{l}\text { Nasal surgery (rhinoplasty, } \\
\text { septoplasty, ESS)/no } \\
\text { cardiovascular disease }\end{array}$ & $\begin{array}{l}\text { Dexmedetomidine vs. } \\
\text { saline } \\
\text { Dexmedetomidine vs. } \\
\text { remifentanil }\end{array}$ & $\begin{array}{l}\text { Postoperative pain } \\
\text { Adverse effects (hypotension, } \\
\text { PONV) }\end{array}$ & Low risk of bias \\
\hline $\begin{array}{r}\text { Kavalci et al. } \\
\text { (2013) [28] }\end{array}$ & $60(34: 26) / 33.03 \pm 12.4$ & $\begin{array}{l}\text { Septoplasty/no } \\
\text { cardiovascular disease }\end{array}$ & $\begin{array}{l}\text { Dexmedetomidine vs. } \\
\text { remifentanil }\end{array}$ & Surgical time & Low risk of bias \\
\hline $\begin{array}{r}\text { Gupta et al. } \\
(2016)[7]\end{array}$ & $40(21: 19) / 34.13 \pm 13.03$ & $\begin{array}{l}\text { ESS/no cardiovascular } \\
\text { disease }\end{array}$ & $\begin{array}{l}\text { Dexmedetomidine vs. } \\
\text { saline }\end{array}$ & $\begin{array}{l}\text { Dose of fentanyl } \\
\text { Postoperative pain } \\
\text { Adverse effects (hypotension, } \\
\text { PONV) }\end{array}$ & Unclear risk of bias \\
\hline $\begin{array}{l}\text { Ozcan et al. } \\
\text { (2012) [29] }\end{array}$ & $50(25: 25) / 36.92 \pm 12.67$ & $\begin{array}{l}\text { ESS/no cardiovascular } \\
\text { disease }\end{array}$ & $\begin{array}{l}\text { Dexmedetomidine vs. } \\
\text { remifentanil }\end{array}$ & $\begin{array}{l}\text { Surgical time } \\
\text { Adverse effects (PONV) }\end{array}$ & High risk of bias \\
\hline $\begin{array}{l}\text { Kumari et al. } \\
\text { (2016) [37] }\end{array}$ & $48(29: 17) / 36.4 \pm 9.7$ & $\begin{array}{l}\text { ESS/no cardiovascular } \\
\text { disease }\end{array}$ & $\begin{array}{l}\text { Dexmedetomidine vs. } \\
\text { saline }\end{array}$ & Operative bleeding & Low risk of bias \\
\hline $\begin{array}{l}\text { Kaur et al. } \\
\text { (2016) [30] }\end{array}$ & $\begin{array}{l}52(\text { Sex ratio not } \\
\quad \text { reported)/36.96 } \pm 14.94\end{array}$ & $\begin{array}{l}\text { ESS/no cardiovascular } \\
\text { disease }\end{array}$ & $\begin{array}{l}\text { Dexmedetomidine vs. } \\
\text { saline }\end{array}$ & $\begin{array}{l}\text { Surgical time } \\
\text { Dose of fentanyl }\end{array}$ & Unclear risk of bias \\
\hline $\begin{array}{c}\text { Khurshid et al. } \\
\text { (2015) [43] }\end{array}$ & $\begin{array}{l}100 \text { (Sex ratio not } \\
\text { reported)/range, 20-60 }\end{array}$ & $\begin{array}{l}\text { Nasal surgery (rhinoplasty, } \\
\text { septoplasty, ESS)/no } \\
\text { cardiovascular disease }\end{array}$ & $\begin{array}{l}\text { Dexmedetomidine vs. } \\
\text { saline }\end{array}$ & Adverse effects (PONV) & Unclear risk of bias \\
\hline $\begin{array}{l}\text { Ayoglu et al. } \\
(2008)[6]\end{array}$ & $40(22: 18) / 34.7 \pm 10.6$ & $\begin{array}{l}\text { ESS/no cardiovascular } \\
\text { disease }\end{array}$ & $\begin{array}{l}\text { Dexmedetomidine vs. } \\
\text { saline }\end{array}$ & $\begin{array}{l}\text { Surgical time } \\
\text { Operative bleeding } \\
\text { Dose of fentanyl }\end{array}$ & Unclear risk of bias \\
\hline $\begin{array}{l}\text { Xu et al. } \\
\text { (2016) [31] }\end{array}$ & $60(40: 20) / 40.2 \pm 11.5$ & $\begin{array}{l}\text { ESS/no cardiovascular } \\
\text { disease }\end{array}$ & $\begin{array}{l}\text { Dexmedetomidine vs. } \\
\text { saline }\end{array}$ & $\begin{array}{l}\text { Surgical time } \\
\text { Adverse effects (PONV) }\end{array}$ & Low risk of bias \\
\hline $\begin{array}{l}\text { Gupta et al. } \\
\text { (2016) [32] }\end{array}$ & $50(30: 20) / 31.2 \pm 6.7$ & $\begin{array}{l}\text { ESS/no cardiovascular } \\
\text { disease }\end{array}$ & $\begin{array}{l}\text { Dexmedetomidine vs. } \\
\text { saline }\end{array}$ & $\begin{array}{l}\text { Surgical time } \\
\text { Dose of fentanyl } \\
\text { Adverse effects (PONV) }\end{array}$ & Unclear risk of bias \\
\hline $\begin{array}{l}\text { Kim et al. } \\
\text { (2013) [33] }\end{array}$ & $\begin{array}{l}100 \text { (72:28)/33 (range, } \\
20-58)\end{array}$ & $\begin{array}{l}\text { Nasal surgery (rhinoplasty, } \\
\text { septoplasty, ESS)/no } \\
\text { cardiovascular disease }\end{array}$ & $\begin{array}{l}\text { Dexmedetomidine vs. } \\
\text { saline }\end{array}$ & $\begin{array}{l}\text { Surgical time } \\
\text { Operative bleeding } \\
\text { Postoperative pain } \\
\text { Adverse effects (hypotension, } \\
\text { PONV) }\end{array}$ & Low risk of bias \\
\hline $\begin{array}{l}\text { Kim et al. } \\
\text { (2015) [3] }\end{array}$ & $39(23: 16) / 39.2 \pm 12.5$ & $\begin{array}{l}\text { ESS/no cardiovascular } \\
\text { disease }\end{array}$ & $\begin{array}{l}\text { Dexmedetomidine vs. } \\
\text { remifentanil }\end{array}$ & $\begin{array}{l}\text { Surgical time } \\
\text { Postoperative pain } \\
\text { Adverse effects (PONV) }\end{array}$ & Low risk of bias \\
\hline $\begin{array}{l}\text { Karabayirli et al. } \\
\text { (2017) [34] }\end{array}$ & $\begin{array}{l}50(26: 24) / 37 \text { (range, } \\
27-46)\end{array}$ & $\begin{array}{l}\text { ESS/no cardiovascular } \\
\text { disease }\end{array}$ & $\begin{array}{l}\text { Dexmedetomidine vs. } \\
\text { remifentanil }\end{array}$ & $\begin{array}{l}\text { Surgical time } \\
\text { Operative bleeding } \\
\text { Surgical field } \\
\text { Adverse effects (PONV) }\end{array}$ & Low risk of bias \\
\hline $\begin{array}{l}\text { Cardesin et al. } \\
\text { (2015) [9] }\end{array}$ & $47(29: 18) / 48.3 \pm 13.4$ & $\begin{array}{l}\text { ESS/no cardiovascular } \\
\text { disease }\end{array}$ & $\begin{array}{l}\text { Clonidine vs. } \\
\text { remifentanil }\end{array}$ & $\begin{array}{l}\text { Surgical time } \\
\text { Operative bleeding }\end{array}$ & Low risk of bias \\
\hline $\begin{array}{l}\text { Jangra et al. } \\
\text { (2016) [12] }\end{array}$ & $20(10: 10) / 36.4 \pm 9.7$ & $\begin{array}{l}\text { ESS/no cardiovascular } \\
\text { disease }\end{array}$ & $\begin{array}{l}\text { Beta-blocker (esmolol) } \\
\text { vs. saline }\end{array}$ & $\begin{array}{l}\text { Surgical time } \\
\text { Operative bleeding }\end{array}$ & Low risk of bias \\
\hline $\begin{array}{l}\text { Tugrul et al. } \\
\text { (2016) [14] }\end{array}$ & $52(28: 24) / 43.2 \pm 8.3$ & $\begin{array}{l}\text { ESS/no cardiovascular } \\
\text { disease }\end{array}$ & Clonidine vs. placebo & $\begin{array}{l}\text { Surgical time } \\
\text { Operative bleeding }\end{array}$ & Low risk of bias \\
\hline $\begin{array}{l}\text { Shams et al. } \\
(2013)[1]\end{array}$ & $\begin{array}{l}40 \text { (Sex ratio not } \\
\text { reported)/36.1 } 18.8\end{array}$ & $\begin{array}{l}\text { ESS/no cardiovascular } \\
\text { disease }\end{array}$ & $\begin{array}{l}\text { Beta-blocker (esmolol) } \\
\text { vs. dexmedetomidine }\end{array}$ & $\begin{array}{l}\text { Surgical time } \\
\text { Operative bleeding } \\
\text { Dose of fentanyl }\end{array}$ & High risk of bias \\
\hline $\begin{array}{l}\text { Jiwanmall et al. } \\
\text { (2017) [36] }\end{array}$ & $\begin{array}{l}60(\text { Sex ratio not } \\
\text { reported)/range, } \\
\text { 18-65 }\end{array}$ & $\begin{array}{l}\text { ESS/no cardiovascular } \\
\text { disease }\end{array}$ & Clonidine vs. placebo & $\begin{array}{l}\text { Surgical time } \\
\text { Postoperative pain } \\
\text { Adverse effects (hypotension) }\end{array}$ & Low risk of bias \\
\hline $\begin{array}{l}\text { Jabalameli et al. } \\
\text { (2005) [15] }\end{array}$ & $113(63: 50) / 43 \pm 12$ & $\begin{array}{l}\text { ESS/no cardiovascular } \\
\text { disease }\end{array}$ & Clonidine vs. placebo & $\begin{array}{l}\text { Surgical time } \\
\text { Operative bleeding } \\
\text { Dose of fentanyl }\end{array}$ & Unclear risk of bias \\
\hline
\end{tabular}


Table 1. Continued

\begin{tabular}{|c|c|c|c|c|c|}
\hline Study & $\begin{array}{c}\text { Patient (male:female)/ } \\
\text { age (yr) }\end{array}$ & $\begin{array}{c}\text { ENT operation/ } \\
\text { underlying disease }\end{array}$ & Comparison & Outcome measure analyzed & $\begin{array}{l}\text { Risk of bias of } \\
\text { randomized studies }\end{array}$ \\
\hline $\begin{array}{l}\text { Mohseni and } \\
\text { Ebneshahidi } \\
\text { (2011) [17] }\end{array}$ & $84(50: 34) / 36 \pm 13$ & $\begin{array}{l}\text { ESS/no cardiovascular } \\
\text { disease }\end{array}$ & Clonidine vs. placebo & $\begin{array}{l}\text { Surgical time } \\
\text { Operative bleeding }\end{array}$ & Low risk of bias \\
\hline $\begin{array}{l}\text { Sajedi et al. } \\
\text { (2016) [22] }\end{array}$ & $62(28 / 34) / 36.36 \pm 12.35$ & $\begin{array}{l}\text { ESS/no cardiovascular } \\
\text { disease }\end{array}$ & $\begin{array}{l}\text { Beta-blocker } \\
\text { (labetalol) vs. } \\
\text { dexmedetomidine }\end{array}$ & $\begin{array}{l}\text { Surgical time } \\
\text { Operative bleeding } \\
\text { Dose of fentanyl } \\
\text { Postoperative pain } \\
\text { Adverse effects (hypotension, } \\
\text { PONV) }\end{array}$ & Unclear risk of bias \\
\hline $\begin{array}{l}\text { Nair et al. } \\
\text { (2004) [25] }\end{array}$ & $80(50: 30) /$ mean, 43.7 & $\begin{array}{l}\text { ESS/no cardiovascular } \\
\text { disease }\end{array}$ & $\begin{array}{l}\text { Beta-blocker } \\
\text { (metoprolol) vs. } \\
\text { placebo }\end{array}$ & $\begin{array}{l}\text { Surgical time } \\
\text { Operative bleeding }\end{array}$ & Low risk of bias \\
\hline $\begin{array}{l}\text { Shen et al. } \\
\text { (2011) [26] }\end{array}$ & $40(31: 9) / 45.1 \pm 3.4$ & $\begin{array}{l}\text { ESS/no cardiovascular } \\
\text { disease }\end{array}$ & $\begin{array}{l}\text { Beta-blocker (esmolol) } \\
\text { vs. placebo }\end{array}$ & $\begin{array}{l}\text { Surgical time } \\
\text { Operative bleeding }\end{array}$ & Low risk of bias \\
\hline $\begin{array}{r}\text { Bayram et al. } \\
(2015)[27]\end{array}$ & $\begin{array}{l}60(\text { Sex ratio not } \\
\quad \text { reported)/range, 18-65 }\end{array}$ & $\begin{array}{l}\text { ESS/no cardiovascular } \\
\text { disease }\end{array}$ & $\begin{array}{l}\text { Placebo vs. } \\
\text { dexmedetomidine }\end{array}$ & $\begin{array}{l}\text { Surgical time } \\
\text { Operative bleeding } \\
\text { Adverse effects (hypotension, } \\
\text { PONV) }\end{array}$ & Unclear risk of bias \\
\hline $\begin{array}{l}\text { Praveen et al. } \\
\text { (2016) [38] }\end{array}$ & 60 (41:19)/mean, 31.43 & $\begin{array}{l}\text { ESS/no cardiovascular } \\
\text { disease }\end{array}$ & $\begin{array}{l}\text { Nitroglycerin vs. } \\
\text { dexmedetomidine }\end{array}$ & $\begin{array}{l}\text { Operative bleeding } \\
\text { Dose of fentanyl }\end{array}$ & Unclear risk of bias \\
\hline $\begin{array}{l}\text { Das et al. } \\
\qquad(2016)[13]\end{array}$ & $60(48: 12) / 38.68 \pm 9.72$ & $\begin{array}{l}\text { ESS/no cardiovascular } \\
\text { disease }\end{array}$ & $\begin{array}{l}\text { Beta-blocker } \\
\text { (esmolol) vs. } \\
\text { dexmedetomidine } \\
\text { vs. clonidine }\end{array}$ & $\begin{array}{l}\text { Surgical time } \\
\text { Operative bleeding } \\
\text { Dose of fentanyl } \\
\text { Adverse effects (hypotension, } \\
\text { PONV) }\end{array}$ & Low risk of bias \\
\hline $\begin{array}{l}\text { Bajwa et al. } \\
\text { (2016) [19] }\end{array}$ & $100(66: 34) / 36.4 \pm 6.1$ & $\begin{array}{l}\text { ESS/no cardiovascular } \\
\text { disease }\end{array}$ & $\begin{array}{l}\text { Nitroglycerine vs. } \\
\text { beta-blocker } \\
\text { (esmolol) vs. } \\
\text { dexmedetomidine }\end{array}$ & $\begin{array}{l}\text { Surgical time } \\
\text { Dose of fentanyl } \\
\text { Adverse effects (PONV) }\end{array}$ & Low risk of bias \\
\hline $\begin{array}{l}\text { Bairy et al. } \\
\text { (2017) [10] }\end{array}$ & $59(26: 33) / 46 \pm 15$ & $\begin{array}{l}\text { ESS/no cardiovascular } \\
\text { disease }\end{array}$ & $\begin{array}{l}\text { Clonidine vs. opioids } \\
\text { (remifentanil) }\end{array}$ & $\begin{array}{l}\text { Surgical time } \\
\text { Postoperative pain } \\
\text { Adverse effects (PONV) }\end{array}$ & Unclear risk of bias \\
\hline $\begin{array}{l}\text { Erdivanli et al. } \\
\text { (2018) [11] }\end{array}$ & $88(60: 28) / 39.3 \pm 10.6$ & $\begin{array}{l}\text { ESS/no cardiovascular } \\
\text { disease }\end{array}$ & $\begin{array}{l}\text { Beta-blocker } \\
\text { (metoprolol) vs. } \\
\text { opioids (remifentanil) }\end{array}$ & $\begin{array}{l}\text { Surgical time } \\
\text { Postoperative pain } \\
\text { Adverse effects (hypotension, } \\
\text { PONV) }\end{array}$ & Low risk of bias \\
\hline $\begin{array}{l}\text { Wawrzyniak et al. } \\
\text { (2013) [16] }\end{array}$ & $44(24 /: 20) / 48.6 \pm 7.7$ & $\begin{array}{l}\text { ESS/no cardiovascular } \\
\text { disease }\end{array}$ & Clonidine vs. placebo & Surgical time & Unclear risk of bias \\
\hline $\begin{array}{l}\text { Alkan et al. } \\
\text { (2019) [18] }\end{array}$ & $\begin{array}{l}60(43: 17) / 39 \text { (range, } \\
18-65)\end{array}$ & $\begin{array}{l}\text { ESS/no cardiovascular } \\
\text { disease }\end{array}$ & $\begin{array}{l}\text { Beta-blocker (esmolol) } \\
\text { vs. opioids } \\
\text { (remifentanil) vs. } \\
\text { nitroglycerin }\end{array}$ & Surgical time & High risk of bias \\
\hline $\begin{array}{l}\text { EL-Shmaa et al. } \\
\text { (2017) [20] }\end{array}$ & $60(25: 35) / 47.6 \pm 8.3$ & $\begin{array}{l}\text { ESS/no cardiovascular } \\
\text { disease }\end{array}$ & $\begin{array}{l}\text { Nitroglycerin vs. } \\
\text { beta-blocker } \\
\text { (labetalol) }\end{array}$ & Surgical time & Low risk of bias \\
\hline $\begin{array}{l}\text { Srivastava et al. } \\
\text { (2013) [21] }\end{array}$ & $52(29: 23) / 36.27 \pm 12.42$ & $\begin{array}{l}\text { ESS/no cardiovascular } \\
\text { disease }\end{array}$ & $\begin{array}{l}\text { Beta-blocker } \\
\text { (esmolol) vs. } \\
\text { nitroglycerin }\end{array}$ & Surgical time & Low risk of bias \\
\hline $\begin{array}{l}\text { Wawrzyniak et al. } \\
\text { (2014) [24] }\end{array}$ & $26(16: 10) / 41.4 \pm 14.4$ & $\begin{array}{l}\text { ESS/no cardiovascular } \\
\text { disease }\end{array}$ & $\begin{array}{l}\text { Clonidine vs. } \\
\text { placebo }\end{array}$ & Surgical time & Low risk of bias \\
\hline $\begin{array}{l}\text { Rahimzadeh et al. } \\
\text { (2012) [41] }\end{array}$ & $88(52: 36) / 25.9 \pm 7$ & $\begin{array}{c}\text { Nasal surgery (rhinoplasty, } \\
\text { septoplasty, ESS)/no } \\
\text { cardiovascular disease }\end{array}$ & $\begin{array}{l}\text { Beta-blocker } \\
\text { (esmolol) vs. } \\
\text { placebo }\end{array}$ & Adverse effects (hypotension) & High risk of bias \\
\hline $\begin{array}{l}\text { Aksu et al. } \\
\text { (2009) [40] }\end{array}$ & $40(25: 15) / 25.05 \pm 9.11$ & $\begin{array}{l}\text { Rhinoplasty/no } \\
\text { cardiovascular disease }\end{array}$ & $\begin{array}{l}\text { Dexmedetomidine vs. } \\
\text { opioids (remifentanil) }\end{array}$ & Adverse effects (hypotension) & Unclear risk of bias \\
\hline
\end{tabular}

Values are presented as mean \pm standard deviation otherwise indicated.

ESS, endoscopic sinus surgery; PONV, postoperative nausea and vomiting. 
ized mean differences and 95\% confidence intervals (95\% CIs) for use across all studies to assess operative time, mean intraoperative bleeding, intraoperative fentanyl dose administered, and postoperative pain. In all other cases, the outcomes of the incidence analysis were assessed using odds ratios. To rank treatment options graphically, the surface under the cumulative ranking curve (SUCRA) and mean ranks were adopted. SUCRA represents the probability of a treatment ranking best [44]. In this study, SUCRA ranged from 0 to 1 , with 1 indicating that the treatment option was statistically best and 0 the worst. Direct and indirect comparisons were adequately homogeneous. A loop-specific approach was used to distinguish heterogeneity in all triangular or quadratic loops in the NMA model [45]. The discrepancy between direct and indirect evidence with the $95 \%$ $\mathrm{CI}$ was used to distinguish heterogeneity in all loops. Heterogeneity was defined as the difference between direct and indirect evidence with a $95 \% \mathrm{CI}$ excluding 0 . The node-splitting model was used to distinguish heterogeneity between the direct and indirect evidence [46]. In addition, we made comparison-adjusted funnel plots to assess potential publication bias [47].

\section{RESULTS}

The literature review yielded 37 studies containing 1945 participants. Table 1 shows the results of the bias assessment and the study characteristics. Clonidine had the greatest effect on operative time compared with placebo $(-0.699 ; 95 \% \mathrm{CI},-0.977$ to $-0.421)$, followed by dexmedetomidine $(-0.466 ; 95 \% \mathrm{CI},-0.705$ to -0.226$)$, opioids ( -0.418 ; $95 \% \mathrm{CI},-0.728$ to -0.108$)$, nitroglycerin $(-0.260 ; 95 \% \mathrm{CI},-0.651$ to 0.132$)$, and beta-blockers $(-0.253 ; 95 \% \mathrm{CI},-0.554$ to 0.048$)$ (Fig. $2 \mathrm{~A})$. In terms of intraoperative fentanyl administration, dexmedetomidine had the greatest effect compared with placebo $(-3.683 ; 95 \% \mathrm{CI},-4.848$ to -2.518$)$, followed by nitroglycerin $(-1.491 ; 95 \% \mathrm{CI},-3.224$ to 0.242$)$, beta-blockers $(-1.039 ; 95 \% \mathrm{CI},-2.739$ to 0.661$)$, and clonidine (-0.417; 95\% CI, -2.022 to 1.188$)$ (Fig. 2B). The greatest effect on intraoperative bleeding compared with placebo was achieved with dexmedetomidine $(-0.971 ; 95 \% \mathrm{CI},-1.161$ to $-0.781)$, followed by nitroglycerin $(-0.945 ; 95 \% \mathrm{CI},-1.420$ to $-0.470)$, opioids $(-0.691 ; 95 \% \mathrm{CI},-0.968$ to -0.414$)$, clonidine $(-0.571 ; 95 \% \mathrm{CI},-0.768$ to -0.374$)$, and beta-blockers $(-0.437$; $95 \% \mathrm{CI},-0.650$ to -0.225 ) (Fig. 2C). The greatest effect on postoperative pain compared with placebo was observed for dexmedetomidine $(-2.065$; 95\% CI, -3.170 to -0.960$)$, followed by beta-blockers $(-1.414 ; 95 \% \mathrm{CI},-3.059$ to 0.232$)$, clonidine $(-1.151 ; 95 \%$ CI, -2.519 to 0.218$)$, and opioids $(-0.764 ; 95 \%$ CI, -2.054 to 0.526 ) (Fig. 2D). Intraoperative and postoperative morbidities were in part attributed to the adverse effects of the hypotensive agents evaluated. The binary outcomes of hypotension and nausea/vomiting showed no significant differences in intraoperative or postoperative morbidities between the treated and control groups (Fig. 2E and F).

An evaluation of whether the direct and indirect comparisons were sufficiently similar in the NMA showed no global inconsistencies regarding intraoperative bleeding $(P=0.255)$, hypotension $(P=0.222)$, nausea and vomiting $(P=0.080)$, pain $(P=0.617)$, intraoperative fentanyl administration $(P=0.151)$, or operative time $(P=0.674)$. Additionally, there were no local inconsistencies among outcomes, except for direct $(-0.217)$ and indirect $(-0.710)$ comparisons of operative time for clonidine. However, this discrepancy only involved the effect size, which may not be meaningful for assessing the effectiveness of a hypotensive agent (Supplementary Tables 4-9).

\section{Results of the ranking hierarchy}

Table 2 shows that dexmedetomidine was superior in terms of pain, intraoperative bleeding, and intraoperative fentanyl administration and ranked second in terms of operative time. There were no statistically significant differences among the tested agents with respect to intraoperative hypotension and postoperative nausea, although dexmedetomidine ranked first and second in postoperative nausea and intraoperative hypotension, respectively. Clonidine ranked first in terms of operative time. Nitroglycerin ranked second in terms of intraoperative bleeding and intraoperative fentanyl administration, but it was not evaluated for hypotension or postoperative pain.

\section{Detection of publication bias}

In all six comparison-adjusted funnel plots (Fig. 3), the scatter plots with the same symbols were visually symmetrical, meaning that publication bias was relatively low for operative time, intraoperative fentanyl administration, intraoperative bleeding, postoperative pain, hypotension, and nausea and vomiting. A linear regression test of funnel plot asymmetry also showed no significant publication bias $(P>0.05)$.

\section{DISCUSSION}

Previous meta-analyses have documented the efficacy of several hypotensive agents in decreasing intraoperative bleeding and improving the surgical view during nasal surgery, especially when performed endoscopically [48-52]. However, the limitations of these studies hindered their clinical relevance, as they consisted of conventional pairwise meta-analyses, which can compare only two treatments, but cannot assess multiple treatments or provide a ranking of their effectiveness [42]. This makes it difficult for clinicians to select the optimal treatment among several treatments demonstrated to be effective in previous meta-analyses.

NMA is a novel analytic approach that enables simultaneous comparisons of multiple interventions. It also allows quantitative comparisons of treatments that previously had not been 

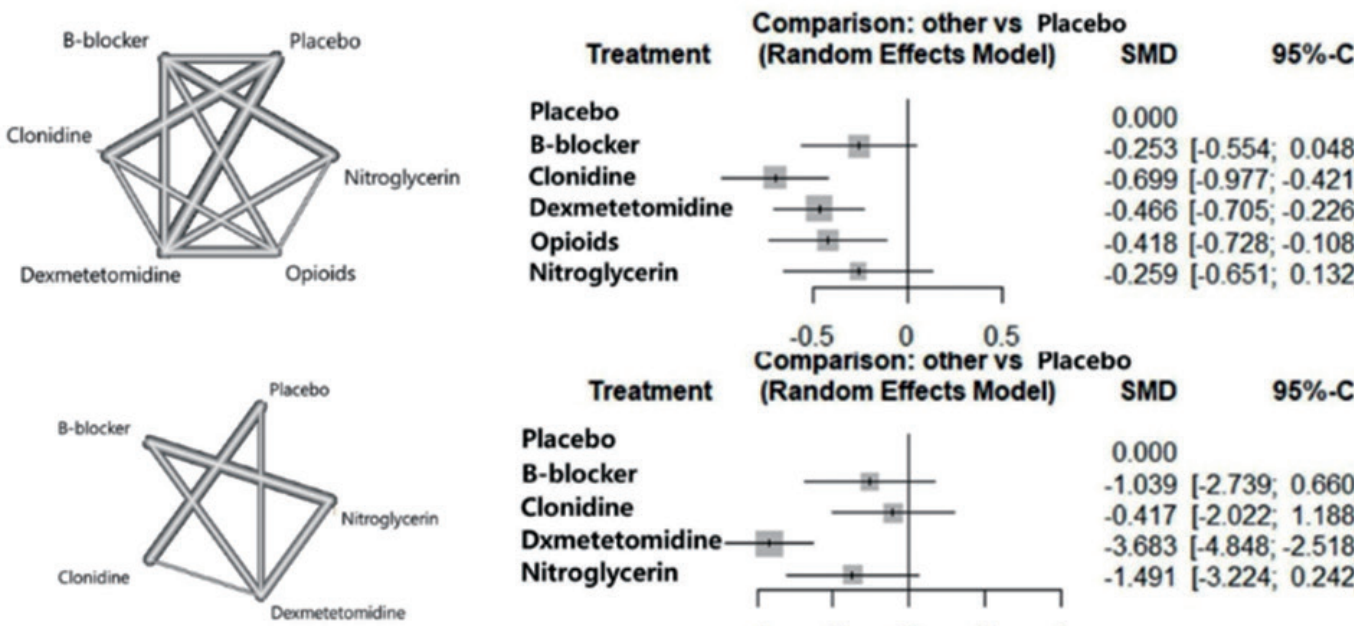

Treatment (Random Effects Model) SMD 95\%-Cl

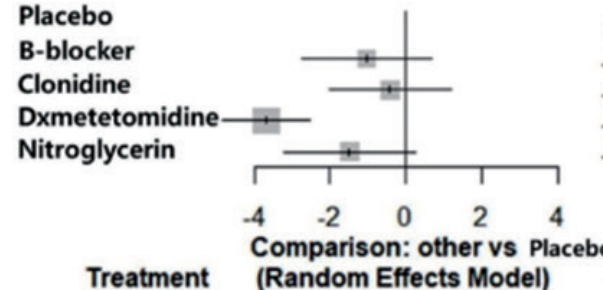

0.000

$-1.039[-2.739 ; 0.660]$

$-0.417[-2.022 ; 1.188]$

$-3.683[-4.848 ;-2.518]$

$-1.491[-3.224 ; 0.242]$

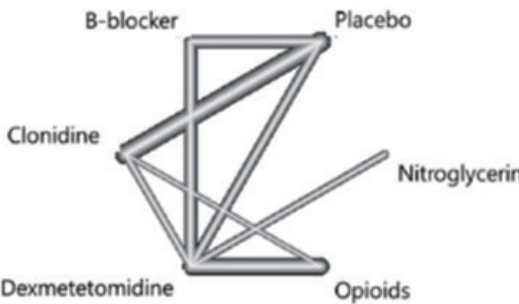

Treatment

(Random Effects Model)

SMD

$95 \% \cdot \mathrm{Cl}$

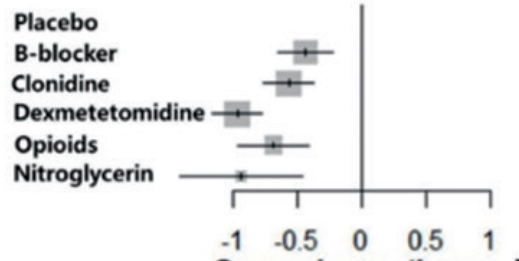

0.000

$-0.437[-0.650 ;-0.225]$

$-0.571[-0.767 ;-0.374]$

$-0.971[-1.161 ;-0.781]$

$-0.691[-0.968 ;-0.414]$

$-0.945[-1.420 ;-0.470]$

B

A

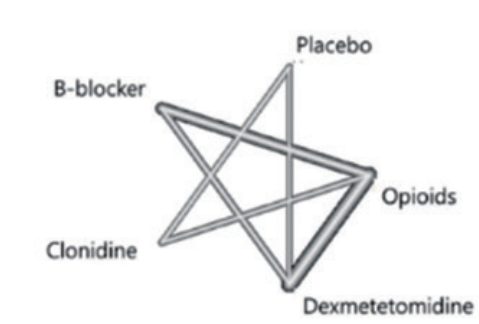

Treatment

Comparison: other vs Placebo

(Random Effects Model)

SMD

$95 \%-\mathrm{Cl}$
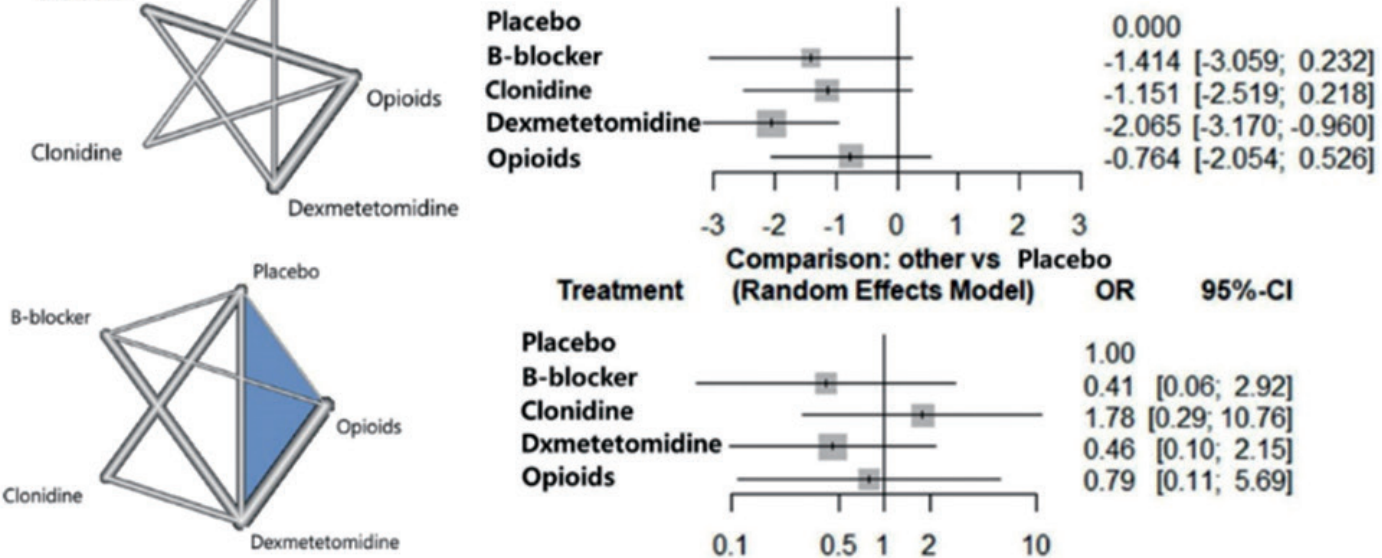

\section{Treatment}

(Random Effects Model)

OR

$95 \%-\mathrm{Cl}$
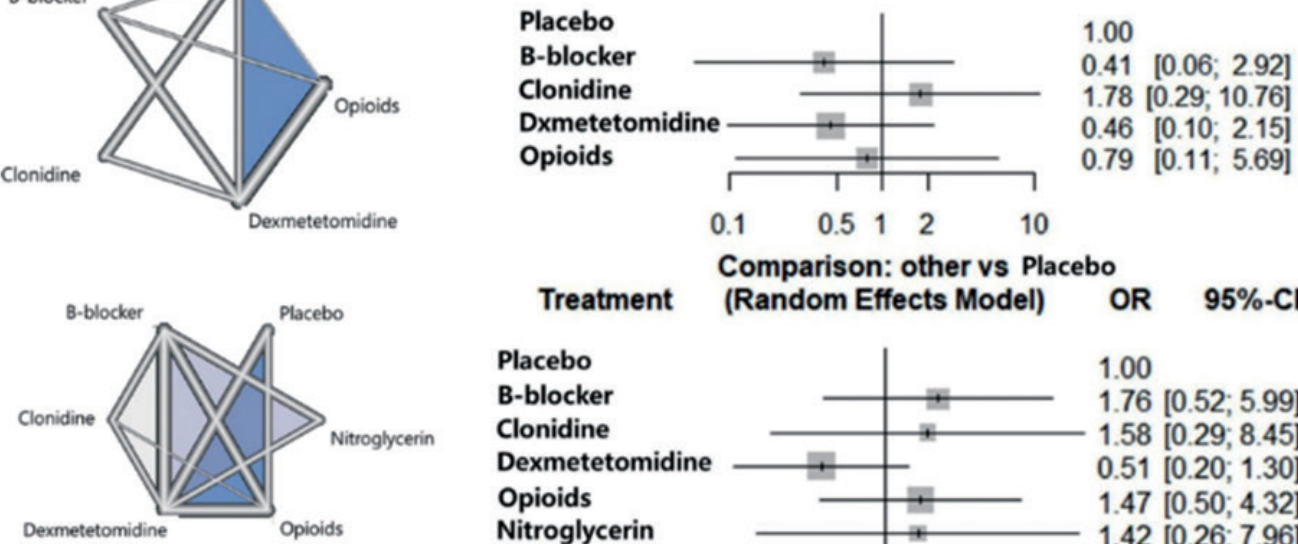

Treatment

Comparison: other vs Placebo

Placebo

B-blocker

Clonidine

Dexmetetomidine

Opioids

Nitroglycerin

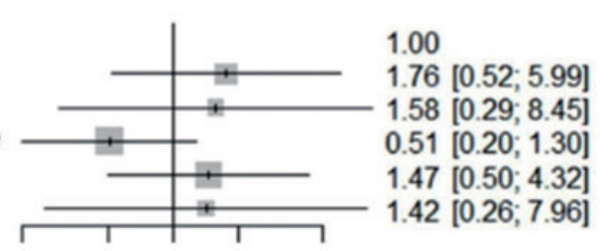

$\begin{array}{lllll}0.2 & 0.5 & 1 & 2 & 5\end{array}$

Fig. 2. Evidence structure of eligible comparisons (left) and forest plots (right) for the network meta-analysis. (A) Operative time, (B) fentanyl administration, (C) intraoperative bleeding, (D) postoperative pain, (E) hypotension, and (F) nausea and vomiting. Lines indicate direct comparisons in the eligible randomized controlled studies (RCTs). The width of the lines represents the number of RCTs for each pairwise comparison. The standardized mean differences and relative risks of intraoperative and postoperative events are shown. SMD, standardized mean difference; $\mathrm{Cl}$, confidence interval; OR, odds ratio. 
Table 2. Ranked probabilities of the effectiveness of different treatments on the incidence of nausea and vomiting, hypotension, postoperative pain, intraoperative bleeding, intraoperative fentanyl administration, and operative time

\begin{tabular}{|c|c|c|c|c|c|c|c|c|c|c|c|c|}
\hline \multirow{2}{*}{ Treatment } & \multicolumn{2}{|c|}{ Nausea } & \multicolumn{2}{|c|}{ Hypotension } & \multicolumn{2}{|l|}{ Pain } & \multicolumn{2}{|c|}{ Blood loss } & \multicolumn{2}{|c|}{ Intraoperative fentanyl } & \multicolumn{2}{|c|}{ Operative time } \\
\hline & SUCRA (\%) & Rank & SUCRA (\%) & Rank & SUCRA (\%) & Rank & SUCRA (\%) & Rank & SUCRA (\%) & Rank & SUCRA (\%) & Rank \\
\hline Placebo & 0.602 & 2 & 0.373 & 4 & 0.055 & 5 & 0 & 6 & 0.117 & 5 & 0.030 & 6 \\
\hline Beta-blocker & 0.273 & 6 & 0.752 & 1 & 0.642 & 2 & 0.247 & 5 & 0.471 & 3 & 0.335 & 5 \\
\hline Clonidine & 0.368 & 5 & 0.173 & 5 & 0.538 & 3 & 0.908 & 1 & 0.100 & 1 & 0.702 & 2 \\
\hline Dexmedetomidine & 0.954 & 1 & 0.749 & 2 & 0.934 & 1 & 0.583 & 3 & - & - & 0.604 & 3 \\
\hline Opioids & 0.383 & 4 & 0.453 & 3 & 0.332 & 4 & 0.842 & 2 & 0.632 & 2 & 0.357 & 4 \\
\hline Nitroglycerin & 0.420 & 3 & - & - & - & - & 0 & 6 & 0.117 & 5 & 0.030 & 6 \\
\hline
\end{tabular}

SUCRA, surface under the cumulative ranking curve.
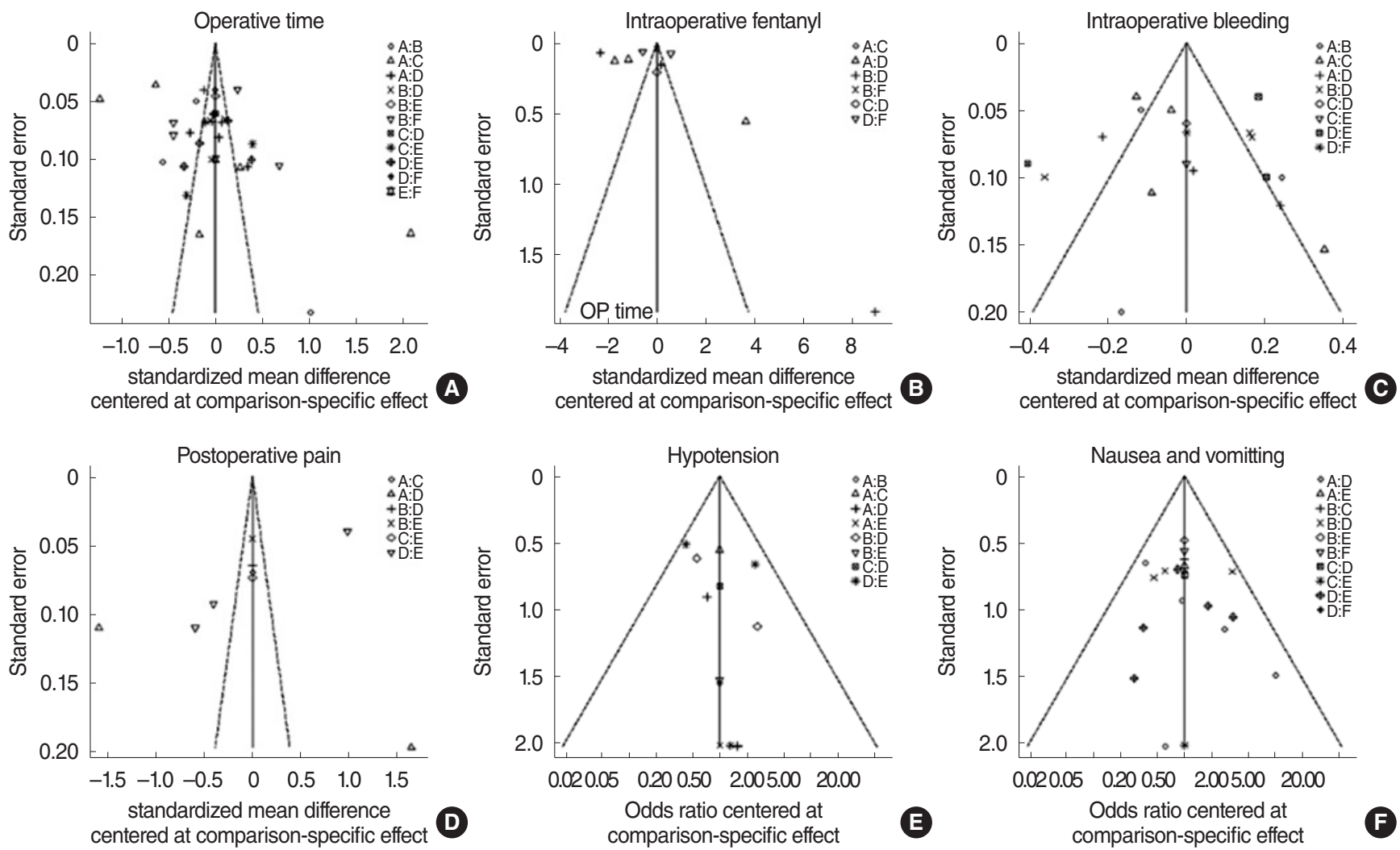

Fig. 3. Funnel plot for publication bias. (A) Placebo. (B) Beta-blocker. (C) Clonidine. (D) Dexmedetomidine. (E) Opioids. (F) Nitroglycerin.

compared directly [53], by using direct and indirect data and combinations of evidence from different dimensions [8]. This study utilized NMA to resolve the limitations of previous studies of nasal surgery that examined single, rather than multiple, treatment options. Specifically, we conducted a systematic review using an NMA to rank the effects of six agents used to induce intraoperative hypotension based on intraoperative and postoperative morbidity endpoints.

Although they have no direct sympathomimetic effects and do not influence vasodilation, opioids, in particular remifentanil, reduce surgical blood loss by effectively lowering heart rate, cardiac output, and blood pressure without the need for other hypotensive agents [35]. Based on recent reports, we defined six intervention groups - placebo, clonidine, dexmedetomidine, beta-blockers, nitroglycerine, and opioids-and constructed treatment networks for the NMA. All of the studied agents showed beneficial effects on reducing intraoperative bleeding, but only dexmedetomidine had positive effects on all of the other examined outcomes (operative time, intraoperative fentanyl administration, and postoperative pain) and ranked highest compared with placebo for all outcomes except operative time. Clonidine had the greatest effect on reducing operative time, but it did not significantly reduce either intraoperative fentanyl administration or postoperative pain. Although none of the hypotensive agents evaluated were associated with significant adverse effects, such as severe hypotension or nausea/vomiting, the best results were 
obtained with dexmedetomidine.

Dexmedetomidine is a highly selective alpha 2-adrenoceptor agonist that reduces intraoperative bleeding by lowering blood pressure, thereby achieving hemodynamic stability [50]. Additionally, dexmedetomidine exerts both sedative and analgesic effects without suppressing the respiratory response [2]. The control of intraoperative bleeding contributes to successful surgery by improving visualization of the operating field. When intraoperative bleeding occurs, surgeons must pause the procedure for bleeding control to restore the surgical view, increasing the operative time [7,31]. This explains the relationship between intraoperative bleeding and operative time in our study and the ability of dexmedetomidine to reduce both.

The stress associated with nasal surgery stimulates the sympathetic nervous system [54]. Opioids have been used to prevent sympathetic stimulation and to provide hemodynamic stability during surgery [55]. However, some studies have shown that opioids also reduce intraoperative bleeding and provide a clear surgical view, while others have reported side effects such as nausea/vomiting, respiratory depression, pruritus, sinus bradycardia, and hypotension [7]. Dexmedetomidine alleviates stressinduced responses and provides relatively stable hemodynamics during surgery [7,54]. Our study found that fentanyl administration was significantly lower in the dexmedetomidine group than in the placebo group. These results demonstrate the efficient sympatholytic and analgesic-sparing properties of dexmedetomidine $[55,56]$.

Postoperative pain related to nasal surgery significantly affects patient recovery and requires early and often aggressive management [57]. Surgical trauma itself stimulates nociception and postoperative pain, but it also induces the release of inflammatory mediators from immune cells and non-neuronal cells, resulting in peripheral sensitization, while the partial peripheral nociceptive stimulus may directly cause central sensitization [57]. Dexmedetomidine inhibits the increased release of inflammatory cytokines and exhibits analgesic effects by acting on alpha-2 receptors within the locus coeruleus and spinal cord [7]. These actions inhibit both peripheral and central sensitization, thereby alleviating postoperative pain. In this study, dexmedetomidine significantly reduced postoperative pain but opioids did not, perhaps because hyperalgesia is one of the side effects of opioids [32]. Thus, the overall results showed that dexmedetomidine provides sufficient adjuvant efficiency for patients under general anesthesia.

Nausea/vomiting and hypotension are common adverse effects of hypotensive drugs. In this study, there was no statistically significant difference in the incidence of perioperative adverse effects between the treatment and control groups. Previous studies have shown that a slow loading dose of dexmedetomidine $(1 \mu \mathrm{g} / \mathrm{kg}$ over 10 minutes and continuous infusion at $0.3-$ $0.7 \mu \mathrm{g} / \mathrm{kg} / \mathrm{hr}$ ) can reduce the development of adverse effects $[7,58]$. The safe administration protocols adopted by the studies included in our NMA explain the absence of a significant difference in the frequency of adverse effects.

Our results suggest that dexmedetomidine use in patients undergoing nasal surgery can improve surgical outcomes without serious side effects. Compared with the other hypotensive agents evaluated in our NMA, dexmedetomidine exhibited similar or superior effects in controlling postoperative pain and blood pressure.

However, it should be noted that some of the included studies enrolled small number of patients or performed comparisons based only on indirect evidence. We were also unable to control for the drug administration protocol. In addition, although obtaining a larger sample size via an NMA could enhance reliability and might be clinically useful [47], NMAs have limitations as a statistical method that only assesses computable and numerical information. In particular, unpredictable and difficult-toquantify variables such as surgeon-related factors or the system of each hospital can affect outcomes [59]. Therefore, the findings of this study that dexmedetomidine showed good results for intraoperative bleeding and that clonidine showed favorable results for operative time might have been affected by unpredictable variables. Thus, care should be taken when interpreting these clinical results.

The results of our NMA showed that the systemic use of dexmedetomidine can decrease surgery-related pain without adverse effects, such as nausea/vomiting and significant hypotension, and thus may decrease the need for postoperative analgesics. However, prior to surgery, clinicians must inform patients about the intraoperative use of hypotensive agents.

\section{CONFLICT OF INTEREST}

No potential conflict of interest relevant to this article was reported.

\section{ACKNOWLEDGMENTS}

The search strategy was constructed by the clinical librarian at Bucheon St. Mary's Hospital.

This research was supported by the Basic Science Research Program through the National Research Foundation of Korea (NRF) funded by the Ministry of Education (2018R1D1A1B07045421), the Bio \& Medical Technology Development Program of the National Research Foundation (NRF) funded by the Ministry of Science \& ICT (2018M3A9E8020856, 2019M3A9H2032424, 2019M3E5D5064110), and the Korea Health Industry Development Institute funded by the Ministry of Health and Welfare (HI14C3228), and the Institute of Clinical Medicine Research of Bucheon St. Mary's Hospital, Research Fund (2017, 2018). This research was also supported by a grant from the E.N.T. 
Fund of the Catholic University of Korea (program years 20172018). The sponsors had no role in the study design, data collection and analysis, decision to publish, or preparation of the manuscript.

\section{ORCID}

$\begin{array}{ll}\text { Do Hyun Kim } & \text { https://orcid.org/0000-0002-9248-5572 } \\ \text { Junuk Lee } & \text { https://orcid.org/0000-0002-5417-9013 } \\ \text { Sung Won Kim } & \text { https://orcid.org/0000-0002-8981-2536 } \\ \text { Se Hwan Hwang } & \text { https://orcid.org/0000-0002-2838-7820 }\end{array}$

\section{AUTHOR CONTRIBUTIONS}

Conceptualization: DHK, SHH. Data curation: DHK, JL. Formal analysis: SWK, SHH. Funding acquisition: DHK, SWK, SHH. Methodology: SHH. Project administration: DHK, SHH. Visualization: JL, SWK, SHH. Writing-original draft: DHK, JL, SWK, SHH.Writing-review \& editing: DHK, JL, SWK, SHH.

\section{SUPPLEMENTARY MATERIALS}

Supplementary materials can be found via https://doi.org/10. 21053/ceo.2020.00584.

\section{REFERENCES}

1. Shams T, El Bahnasawe NS, Abu-Samra M, El-Masry R. Induced hypotension for functional endoscopic sinus surgery: a comparative study of dexmedetomidine versus esmolol. Saudi J Anaesth. 2013 Apr;7(2):175-80.

2. Lee K, Yoo BH, Yon JH, Kim KM, Kim MC, Lee WY, et al. General anesthesia versus monitored anesthetic care with dexmedetomidine for closed reduction of nasal bone fracture. Korean J Anesthesiol. 2013 Sep;65(3):209-14.

3. Kim H, Ha SH, Kim CH, Lee SH, Choi SH. Efficacy of intraoperative dexmedetomidine infusion on visualization of the surgical field in endoscopic sinus surgery. Korean J Anesthesiol. 2015 Oct;68(5): 449-54.

4. Bunker JP. Anesthetic effects on surgical blood loss. Ann N Y Acad Sci. 1964 Jul;115:418-21.

5. Akata T. General anesthetics and vascular smooth muscle: direct actions of general anesthetics on cellular mechanisms regulating vascular tone.Anesthesiology. 2007 Feb;106(2):365-91.

6. Ayoglu H, Yapakci O, Ugur MB, Uzun L, Altunkaya H, OzerY, et al. Effectiveness of dexmedetomidine in reducing bleeding during septoplasty and tympanoplasty operations. J Clin Anesth. 2008 Sep; 20(6):437-41.

7. Gupta P, Choudhary R, Ojha D, Jethava D. Dexmedetomidine as an adjuvant for hypotensive anaesthesia during functional endoscopic sinus surgery (FESS). IOSR Dental Med Sci. 2016 Aug;15(8):143-6.

8. Rouse B, Chaimani A, Li T. Network meta-analysis: an introduction for clinicians. Intern Emerg Med. 2017 Feb;12(1):103-11.
9. Cardesin A, Pontes C, Rosell R, Escamilla Y, Marco J, Escobar MJ, et al. A randomised double blind clinical trial to compare surgical field bleeding during endoscopic sinus surgery with clonidine-based or remifentanil-based hypotensive anaesthesia. Rhinology. 2015 Jun; 53(2):107-15.

10. Bairy L, Vanderstichelen M, Jamart J, Collard E. Clonidine or remifentanil for adequate surgical conditions in patients undergoing endoscopic sinus surgery: a randomized study. PeerJ. 2017 May;5:e3370.

11. Erdivanli B, Erdivanli OC, Sen A, Ozdemir A, Tugcugil E, Dursun E. Comparison of metoprolol and tramadol with remifentanil in endoscopic sinus surgery: a randomised controlled trial. Turk J Anaesthesiol Reanim. 2018 Dec;46(6):424-33.

12. Jangra K, Malhotra SK, Gupta A, Arora S. Comparison of quality of the surgical field after controlled hypotension using esmolol and magnesium sulfate during endoscopic sinus surgery. J Anaesthesiol Clin Pharmacol. 2016 Jul-Sep;32(3):325-8.

13. Das A, Chhaule S, Bhattacharya S, Basunia SR, Mitra T, Halder PS, et al. Controlled hypotension in day care functional endoscopic sinus surgery: a comparison between esmolol and dexmedetomidine: a prospective, double-blind, and randomized study. Saudi J Anaesth. 2016 Jul-Sep;10(3):276-82.

14. Tugrul S, Dogan R, Senturk E, Kocak I, Sezen S, Bakan M, et al. Effect of the premedication with oral clonidine on surgical comfort in patients undergoing fess due to advanced nasal polyposis: a randomized double blind clinical trial. Am J Otolaryngol. 2016 Nov-Dec; 37(6):538-43.

15. Jabalameli M, Hashemi M, Soltani H, Hashemi J. Oral clonidine premedication decreases intraoperative bleeding in patients undergoing endoscopic sinus surgery. J Res Med Sci. 2005 Jan;1:25-30.

16. Wawrzyniak K, Kusza K, Cywinski JB, Burduk PK, KazmierczakW. Premedication with clonidine before TIVA optimizes surgical field visualization and shortens duration of endoscopic sinus surgery: results of a clinical trial. Rhinology. 2013 Sep;51(3):259-64.

17. Mohseni M, Ebneshahidi A. The effect of oral clonidine premedication on blood loss and the quality of the surgical field during endoscopic sinus surgery: a placebo-controlled clinical trial. J Anesth. 2011 Aug;25(4):614-7.

18. Alkan A, Honca M, Alkan A, Gulec H, Horasanli E. The efficacy of esmolol, remifentanil and nitroglycerin in controlled hypotension for functional endoscopic sinus surgery. Braz J Otorhinolaryngol. 2019 Oct:S1808-8694(18):30493-2.

19. Bajwa SJ, Kaur J, Kulshrestha A, Haldar R, Sethi R, Singh A. Nitroglycerine, esmolol and dexmedetomidine for induced hypotension during functional endoscopic sinus surgery: a comparative evaluation. J Anaesthesiol Clin Pharmacol. 2016 Apr-Jun;32(2):192-7.

20. El-Shmaa NS, Ezz HA, Younes A. The efficacy of Labetalol versus nitroglycerin for induction of controlled hypotension during sinus endoscopic surgery: a prospective, double-blind and randomized study. J Clin Anesth. 2017 Jun;39:154-8.

21. Srivastava U, Dupargude AB, Kumar D, Joshi K, Gupta A. Controlled hypotension for functional endoscopic sinus surgery: comparison of esmolol and nitroglycerine. Indian J Otolaryngol Head Neck Surg. 2013 Aug;65(Suppl 2):440-4.

22. Sajedi P, Rahimian A, Khalili G. Comparative evaluation between two methods of induced hypotension with infusion of remifentanil and labetalol during sinus endoscopy. J Res Pharm Pract. 2016 OctDec;5(4):264-71.

23. Cardesin A, Pontes C, Rosell R, Escamilla Y, Marco J, Escobar MJ, et al. Hypotensive anaesthesia and bleeding during endoscopic sinus surgery: an observational study. Eur Arch Otorhinolaryngol. 2014 Jun;271(6):1505-11.

24. Wawrzyniak K, Burduk PK, Cywinski JB, Kusza K, KazmierczakW. Improved quality of surgical field during endoscopic sinus surgery after clonidine premedication--a pilot study. Int Forum Allergy Rhi- 
nol. 2014 Jul;4(7):542-7.

25. Nair S, Collins M, Hung P, Rees G, Close D, Wormald PJ. The effect of beta-blocker premedication on the surgical field during endoscopic sinus surgery. Laryngoscope. 2004 Jun;114(6):1042-6.

26. Shen PH,Weitzel EK, Lai JT,Wormald PJ, Ho CS. Intravenous esmolol infusion improves surgical fields during sevoflurane-anesthetized endoscopic sinus surgery: a double-blind, randomized, placebo-controlled trial.Am J Rhinol Allergy. 2011 Nov-Dec;25(6):e208-11.

27. Bayram A, Ulgey A, Gunes I, Ketenci I, Capar A, Esmaoglu A, et al. Comparison between magnesium sulfate and dexmedetomidine in controlled hypotension during functional endoscopic sinus surgery. Rev Bras Anestesiol. 2015 Jan-Feb;65(1):61-7.

28. Kavalci G, Ethemoglu FB, Durukan P, Batuman A, Emre C. Comparison of the effects of dexmedetomidine and remiphentanyl on emergence agitation after sevoflurane anesthesia in adults undergoing septoplasty operation: a randomized double-blind trial. Eur Rev Med Pharmacol Sci. 2013 Nov;17(22):3019-23.

29. Ozcan AA, Ozyurt Y, Saracoglu A, Erkal H, Suslu H, Arslan G, et al. Dexmedetomidine versus remifentanil for controlled hypotensive anesthesia in functional endoscopic sinus surgery. Turk J Anesth Reanim. 2012 Jul;40(5):257-61.

30. Kaur H, Tiwari RL, Bhargava J, Kasliwal N. Effect of dexmedetomidine on consumption of anesthetic agents, duration of surgery, time to extubation and post-operative emergence during endoscopic nasal surgeries: a pilot study. Scholars J Appl Med Sci. 2016 Apr;4(6E): 2180-6.

31. Xu K, Pan Y, Zhu M. Effects of dexmedetomidine on the recovery profiles from general anesthesia in patients undergoing endoscopic sinus surgery. Int J Clin Exp Med. 2016 Sep;9(5):8405-10.

32. Gupta K, Gupta PK, Bhatia KS, Rastogi B, Pandey MN, Agarwal S. Efficacy of dexmedetomidine as an anesthetic adjuvant for functional endoscopic sinus surgery under general anesthesia: a randomizedcontrolled study.Ain-Shams J Anaesthesiol. 2016 Sep;9(2):207-11.

33. Kim SY, Kim JM, Lee JH, Song BM, Koo BN. Efficacy of intraoperative dexmedetomidine infusion on emergence agitation and quality of recovery after nasal surgery. Br J Anaesth. 2013 Aug;111(2):222-8.

34. Karabayirli S, Ugur KS, Demircioglu RI, Muslu B, Usta B, Sert H, et al. Surgical conditions during FESS; comparison of dexmedetomidine and remifentanil. Eur Arch Otorhinolaryngol. 2017 Jan;274(1): 239-45.

35. Lee J, Kim Y, Park C, JeonY, Kim D, Joo J, et al. Comparison between dexmedetomidine and remifentanil for controlled hypotension and recovery in endoscopic sinus surgery. Ann Otol Rhinol Laryngol. 2013 Jul;122(7):421-6.

36. Jiwanmall M, Joselyn AS, Kandasamy S. Intravenous clonidine as a part of balanced anaesthesia for controlled hypotension in functional endoscopic sinus surgery: a randomised controled trial. Indian J Anaesth. 2017 May;61(5):418-23.

37. Kumari I, Sharma S, Surendran K, Naithani U, Saxena SS, Yadav R. Effect of dexmedetomidine on bleeding during endoscopic nasal surgery: a double blind, randomized, clinical study. Eur J Pharm Med Res. 2016 Mar;3(12):290-4.

38. Praveen DV, Pushparani A, Anand K, Sundaraperumal B, Umadevi. Comparison of dexmedetomidine with nitroglycerinefor hypotensive anaesthesia in. Indian J Clin Anesth. 2016 May;3(3):376-88.

39. Polat R, Peker K, Baran I, Bumin Aydın G, Topcu Guloksuz C, Donmez A. Comparison between dexmedetomidine and remifentanil infusion in emergence agitation during recovery after nasal surgery: A randomized double-blind trial. Anaesthesist. 2015 Oct;64(10): 740-6.

40. Aksu R, Akin A, Bicer C, Esmaoglu A, Tosun Z, Boyaci A. Comparison of the effects of dexmedetomidine versus fentanyl on airway reflexes and hemodynamic responses to tracheal extubation during rhinoplasty: A double-blind, randomized, controlled study. CurrTher
Res Clin Exp. 2009 Jun;70(3):209-20.

41. Rahimzadeh P, Faiz SH, Alebouyeh MR. Effects of premedication with metoprolol on bleeding and induced hypotension in nasal surgery. Anesth Pain Med. 2012Winter;1(3):157-61.

42. Shim S, Yoon BH, Shin IS, Bae JM. Network meta-analysis: application and practice using Stata. Epidemiol Health. 2017 Oct;39:e2017047.

43. Khurshid DH, Muneer DK, Sadiq Malla M. Effect of dexmedetomidine on emergence agitation after nasal surgeries. Med Sci Clin Res. 2015;3(9):7527-31.

44. Salanti G, Ades AE, Ioannidis JP. Graphical methods and numerical summaries for presenting results from multiple-treatment meta-analysis: an overview and tutorial. J Clin Epidemiol. 2011 Feb;64(2):16371.

45. Higgins JP, Jackson D, Barrett JK, Lu G, Ades AE, White IR. Consistency and inconsistency in network meta-analysis: concepts and models for multi-arm studies. Res Synth Methods. 2012 Jun;3(2): 98-110.

46. van Valkenhoef G, Dias S, Ades AE, Welton NJ. Automated generation of node-splitting models for assessment of inconsistency in network meta-analysis. Res Synth Methods. 2016 Mar;7(1):80-93.

47. Flather MD, Farkouh ME, Pogue JM, Yusuf S. Strengths and limitations of meta-analysis: larger studies may be more reliable. Control Clin Trials. 1997 Dec;18(6):568-79.

48. Lee HS, Yoon HY, Jin HJ, Hwang SH. Can dexmedetomidine influence recovery profiles from general anesthesia in nasal surgery? Otolaryngol Head Neck Surg. 2018 Jan;158(1):43-53.

49. Quijada-Manuitt MA, Escamilla Y, Vallano A, Cardesin A, BernalSprekelsen M, Pontes C. Use of $\alpha<$ sub $>2</$ sub $>$-adrenergic agonists to improve surgical field visibility in endoscopy sinus surgery: a systematic review of randomized controlled trials. Clin Ther. 2018 Jan; 40(1):136-49.e19.

50. Snidvongs K, TingthanathikulW, Aeumjaturapat S, Chusakul S. Dexmedetomidine improves the quality of the operative field for functional endoscopic sinus surgery: systematic review. J Laryngol Otol. 2015 Jul;129 Suppl 3:S8-13.

51. Amorocho MC, Fat I. Anesthetic techniques in endoscopic sinus and skull base surgery. Otolaryngol Clin North Am. 2016 Jun;49(3):53147.

52. Baker AR, Baker AB. Anaesthesia for endoscopic sinus surgery. Acta Anaesthesiol Scand. 2010 Aug;54(7):795-803.

53. Kanters S, Ford N, Druyts E, Thorlund K, Mills EJ, Bansback N. Use of network meta-analysis in clinical guidelines. Bull World Health Organ. 2016 Oct;94(10):782-4.

54. Bhagat N, Yunus M, Karim HM, Hajong R, Bhattacharyya P, Singh M. Dexmedetomidine in attenuation of haemodynamic response and dose sparing effect on opioid and anaesthetic agents in patients undergoing laparoscopic cholecystectomy: a randomized study. J Clin Diagn Res. 2016 Nov;10(11):UC01-5.

55. Manne GR, Upadhyay MR, SwadiaV. Effects of low dose dexmedetomidine infusion on haemodynamic stress response, sedation and postoperative analgesia requirement in patients undergoing laparoscopic cholecystectomy. Indian J Anaesth. 2014 Nov-Dec;58(6):726-31.

56. Khan ZP, Munday IT, Jones RM, Thornton C, MantTG, Amin D. Effects of dexmedetomidine on isoflurane requirements in healthy volunteers. 1: Pharmacodynamic and pharmacokinetic interactions. Br J Anaesth. 1999 Sep;83(3):372-80.

57. Tang C, Huang X, Kang F, Chai X, Wang S, Yin G, et al. Intranasal dexmedetomidine on stress hormones, inflammatory markers, and postoperative analgesia after functional endoscopic sinus surgery. Mediators Inflamm. 2015;2015:939431.

58. Lee SK. Clinical use of dexmedetomidine in monitored anesthesia care. Korean J Anesthesiol. 2011 Dec;61(6):451-2.

59. Bailar JC 3rd. The promise and problems of meta-analysis. N Engl J Med. 1997 Aug;337(8):559-61. 